\title{
Gastrointestinal cancer diagnostics by terahertz time domain spectroscopy
}

\author{
Anna Goryachuk, Anna Simonova, \\ Mikhail Khodzitsky \\ International laboratory "Radiation medicine" \\ ITMO University \\ Saint Petersburg, Russia \\ goryachuk-anna@yandex.ru
}

\author{
Mariia Borovkova \\ Faculty of Information Technology and Electrical \\ Engineering, University of Oulu, \\ Oulu, Finland \\ International laboratory "Radiation medicine" \\ ITMO University \\ Saint Petersburg, Russia
}

\author{
Abdo Khamid \\ Pavlov First Saint Petersburg State Medical University \\ Saint Petersburg, Russia
}

\begin{abstract}
Samples of fresh excised tissues obtained from patients who had undergone gastric cancer have been investigated. Samples consisted of cancer zone, normal zone and pathologically changed zone. Their optical properties and spectral features were investigated by terahertz time-domain spectroscopy (THz-TDS) in reflection mode. It was found that waveforms of reflected signals from normal and cancer tissues and their optical properties were well distinguished, so it can be concluded that it is possible to discriminate gastric cancer tissue from normal by using THz-TDS.
\end{abstract}

Keywords-THz spectroscopy; biological objects; biophotonics; oncology

\section{INTRODUCTION}

At the present day cancer diseases are the most important problem of medicine which requires effective methods of diagnostic and treatment. During the last decade, the percentage of people who had undergone different kinds of cancer increased by $15.2 \%$ in Russia [1]. There were an estimated 14.1 million new cancer cases around the world in 2012 with 8.2 million cancer deaths [2].

Cancer starts when cells in the body begin to grow out of control. Cells in nearly any part of the body can become cancer, and can spread to other areas of the body. That is why it is important to find an appropriate method to diagnose cancer until it having spread through the whole body.

The most common cancers worldwide in 2016 were projected to be breast cancer, lung and bronchus cancer, prostate cancer, colon and rectum cancer, bladder cancer, melanoma of the skin. The majority of cancer cases occur in Africa, Asia and Central and South America. It is expected that the number of new cancer cases will rise within the next two decades [3].

$\mathrm{THz}$ spectroscopy is expected to become a new modality of biomedical analysis as a noninvasive diagnosis of cancer. Terahertz waves refer to electromagnetic radiation in the frequency range of $0.1-10 \mathrm{THz}$. In the electromagnetic spectrum $\mathrm{THz}$ waves occupy the region between microwaves

Government of Russian Federation grant No 074-U01, Marie Skłodowska-Curie grant agreement No 713606. and infrared. Until about two decades ago the $\mathrm{THz}$ spectrum remained unexplored and unutilized because of lack an appropriate sources and detectors. The development of ultrafast lasers and derivative techniques such as photoconductive switching and sampling in 1980s made it feasible to study the $\mathrm{THz}$ waves.

Over the past two decades when technology for generating and detecting terahertz $(\mathrm{THz})$ radiation has advanced, considerably many fields to investigate were discovered for $\mathrm{THz}$ frequency range. Nowadays terahertz time-domain spectroscopy (THz-TDS) and imaging techniques are applied in various fields such as astronomy, security screening, communications, genetic engineering, pharmaceutical quality control, medical imaging and biomedical engineering [4-5].

Several unique features make $\mathrm{THz}$ very suitable for medical applications. The main feature of $\mathrm{THz}$ spectroscopy which is really important for biophotonics is that the characteristic energies of molecule's rotational and vibrational motions lay in $\mathrm{THz}$ frequency region. Therefore, many chemical and biological molecules can be identified by their characteristic resonant peaks. Moreover, $\mathrm{THz}$ radiation is very sensitive to water [6] so it could provide a high contrast of samples with uneven water content. In addition, $\mathrm{THz}$ radiation is nonionizing because it has very low photon energy and could be applied to in vivo diagnostics. These features are the big advantages for biological structures, because it means that $\mathrm{THz}$ radiation is not harmful for biological samples.

Terahertz (THz) reflectometry has been applied to many biomedical investigations [7] and when endoscope was developed it became possible to extend field of application and investigate internal organs [8]. Therefore, during the past few years, the basic tissue characteristics of each internal organ in $\mathrm{THz}$ frequency regime have been investigated. Besides all applications of $\mathrm{THz}$ radiation in medical research, the main field of interest has become cancer diagnosis as the most dangerous disease for human health. Although advanced gastric cancers have shown poor prognosis [9], the early staged gastric 
cancers can be cured by surgery. For the surgery efficiency, a good operator-independent technique of cancer tissues diagnostics around tumors is needed [10].

$\mathrm{THz}$ spectroscopy and imaging systems can help to detect the early cancer before it is visible or sensitive to any other identification techniques. The latest investigations of examining optical properties in $\mathrm{THz}$ frequency range of skin, breast and colon cancer tissues [11-14] discovered that refractive index and absorption coefficient of tumor tissue are higher than those in a normal tissue. Such distinction is possible due to the fact that $\mathrm{THz}$ radiation is strongly absorbed by water, whereas oncological tissues have increased water content [15] along with structural changes compared with normal tissues.

The aim of the study was to evaluate the feasibility of $\mathrm{THz}$ time-domain reflectometry for the discrimination of gastrointestinal cancer tissues from normal tissues and develop the appropriate method for in vivo discrimination of unique spectral features and optical properties of gastrointestinal tract cancer tumors during surgeries. In this study samples of fresh excised tissues obtained from eight patients suffering from gastric cancer have been investigated. Samples consisted of cancer zone and normal zone, also the pathologically changed epithelium between the zones has been examined to clearly show the distinction of these three tissues.

Waveforms of signals reflected from the fresh tissues were obtained by the THz-TDS in reflection mode. In addition, several optical properties extracted from the $\mathrm{THz}$ reflection signals were analyzed to quantitatively distinguish gastric cancer tissues from normal regions.

\section{SAMPLE PREPARATION}

Six patients were enrolled in the study. Samples of tumors were put into opaque box for fixation.

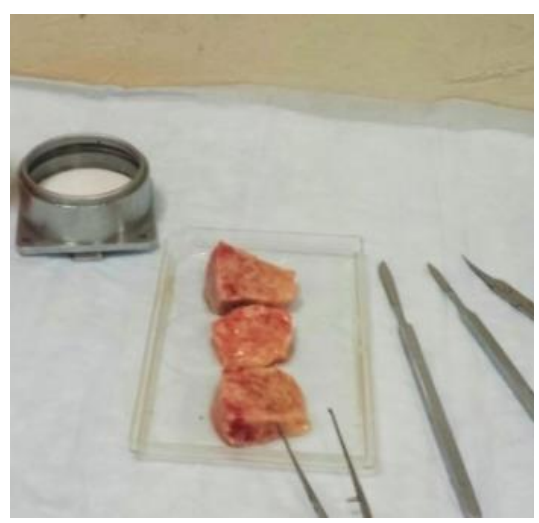

Fig. 1 Photo of sample preparation. All of the specimens consisted of cancer zone, normal zone and pathologically changed zone

Tissue samples were excised during surgeries on the operating table. Samples consisted of tumor tissue, not visually altered tissue and normal tissue. Samples volume were about $3 \times 3.5$ $\times 1 \mathrm{~cm}^{3}$. Specimens were carried in sterile containers filled with a solution of $0.9 \% \mathrm{NaCl}$. Experiments were held within 4 hours after samples were excised.

\section{EXPERIMENTAL SETUP}

Samples were investigated by THz - TD spectrometer in the reflection mode (Fig. 2). The characteristics of the spectrometer are following: the spectral resolution of $15 \mathrm{GHz}$, the sensitivity of lock-in amplifier of $1 \mathrm{mV}$, the lock-in amplifier time constant of $1 \mathrm{~s}$. The $\mathrm{THz}$ broadband pulsed radiation had the following parameters: the spectral range of $0.1-1.5 \mathrm{THz}$, the pulse duration of $2.7 \mathrm{ps}$, the average power of $30 \mu \mathrm{W}$, the average power density $60 \mu \mathrm{W} / \mathrm{cm}^{2}$, THz radiation was generated by femtosecond laser (Yb:KYW) irradiation of undoped indium arsenide crystal. The femtosecond laser parameters are following: the wavelength of $1040 \mathrm{~nm}$, the pulse duration of $120 \mathrm{fs}$, the pulse repetition rate of $75 \mathrm{MHz}$, the power of $1 \mathrm{~W}$.

First, the reference signal of silica window was acquired. Silica window has well-known optical properties, so it is a good material for normalizing of object signal. Then samples were placed under the silica window to acquire the object signal. Waveforms of reference and object reflected signals with both amplitude and phase information were recorded by balanced photodiodes and pictured at PC. So at the stage of waveforms acquisition, the reflected signals amplitudes altered in accordance to the water content.

\section{DATA PRocessing}

The optical properties of examined tissues were calculated using the Matlab Software. The waveforms obtained in the experiments were transformed into frequency domain using Fourier Transform with a Gaussian window function. Dispersions of optical properties (refractive index, permittivity, absorption coefficient) of the examined samples were extracted from the obtained spectra using Fresnel equations [16].

\section{RESUlts}

In this study, optical properties and spectral features of oncological, normal and mixed of oncological and normal epithelium (transitional) tissues were calculated and averaged between 6 specimens. Waveforms of reflected signals from oncological, mixed and normal tissues as well as from distilled water were compared. The Fig. 3 shows amplitude of reflected signals of oncological, mixed and normal tissue. The amplitude of signal of oncological tissue is substantially lower than that of the normal tissue and similar to reflected signal from water. This effect appeared due to strong absorption of $\mathrm{THz}$ radiation by water molecules. Such result approves the theory [15] that the more advanced a tumor becomes the more bodily fluids it contains.

For more accurate discrimination of these three tissues, it is necessary to compare the basic optical properties of tissues such as refractive indices, permittivities and absorption coefficients. The dispersions of optical properties of investigated tissues are shown in Fig. 4-6.

The refractive index dispersion is about $1.7-1.8$ for normal tissues, $2.4-2.5$ for cancer tissues, $1.8-2.2$ for mixed tissues and $2.5-2.6$ for water in the frequency range of $0.4-0.5 \mathrm{THz}$ (Fig. 4). In addition, the averaged permittivities through six experiments with error bars are shown (Fig. 5). Therefore, the comparison of refractive indices could be an additional approach in the diagnostics of gastrointestinal cancer. 


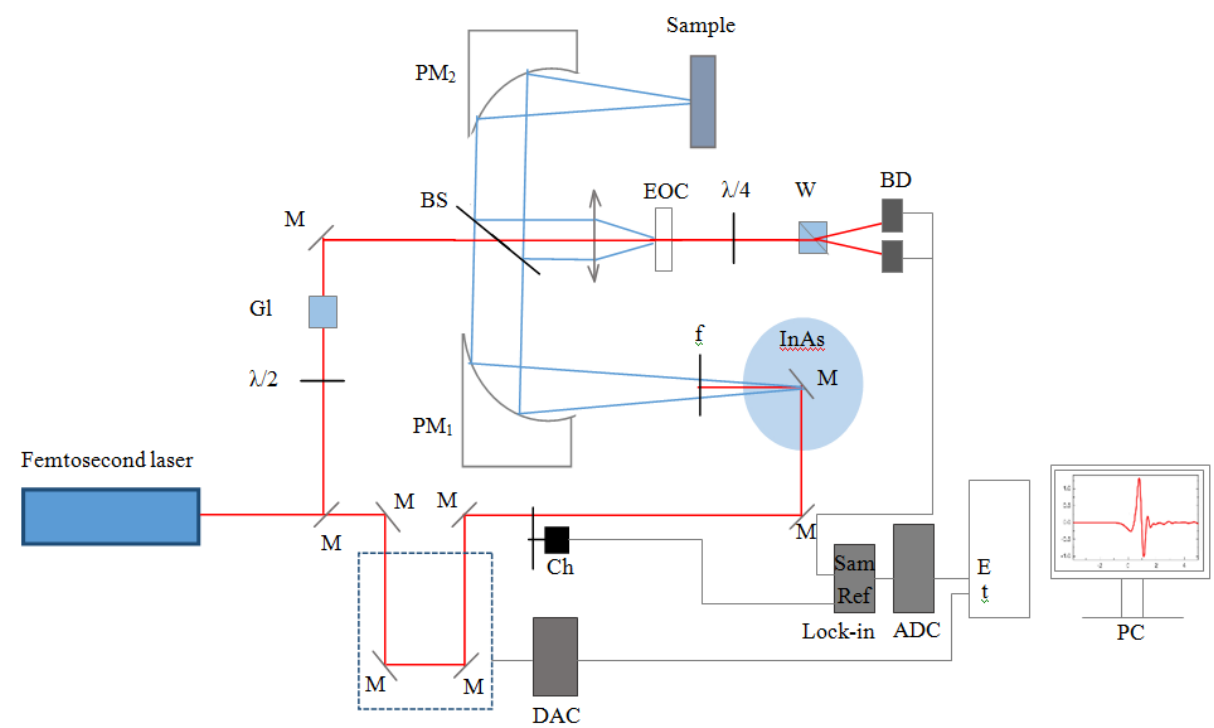

Fig. $2 \mathrm{THz}$ time domain spectrometer in the reflection mode. Femtosecond laser — infrared laser; M — mirrors; BS — beam splitter; InAs — THz radiation generator: crystal of InAs in a magnetic system; PM — parabolic mirrors; Ch — chopper; Sample — biological object; EOC — electro optical crystal of CdTe; $\lambda / 4$ - achromatic quarter-wave plate; $\lambda / 2$ - achromatic half-wave plate; $\mathrm{Gl}$ - Glan prism; W — Wollaston prism; $\mathrm{BD}$ - balanced photodiodes; ADC — analog - digital converter; Lock-in — signal amplifier; DAC — digital - analog converter; PC - personal computer

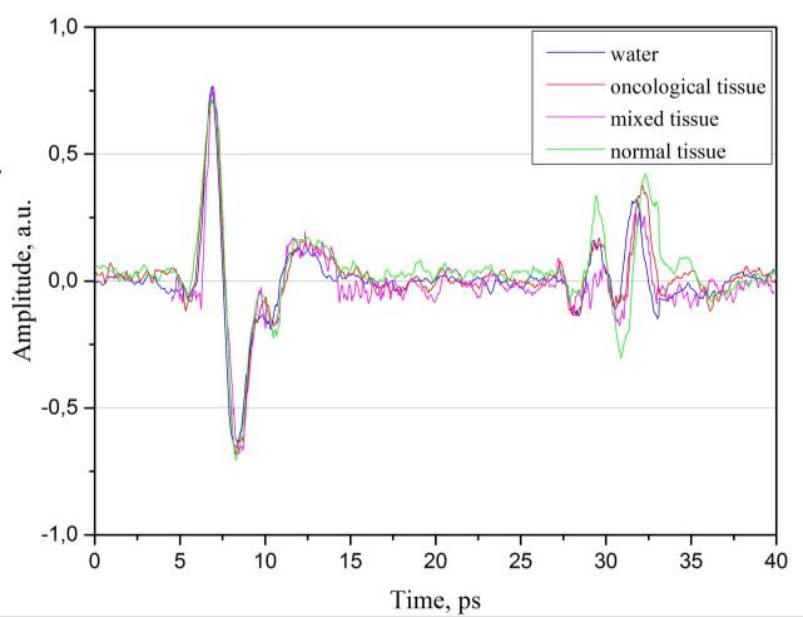

Fig. 3. Waveforms of reflected signals from cancer, mixed, normal tissues and water

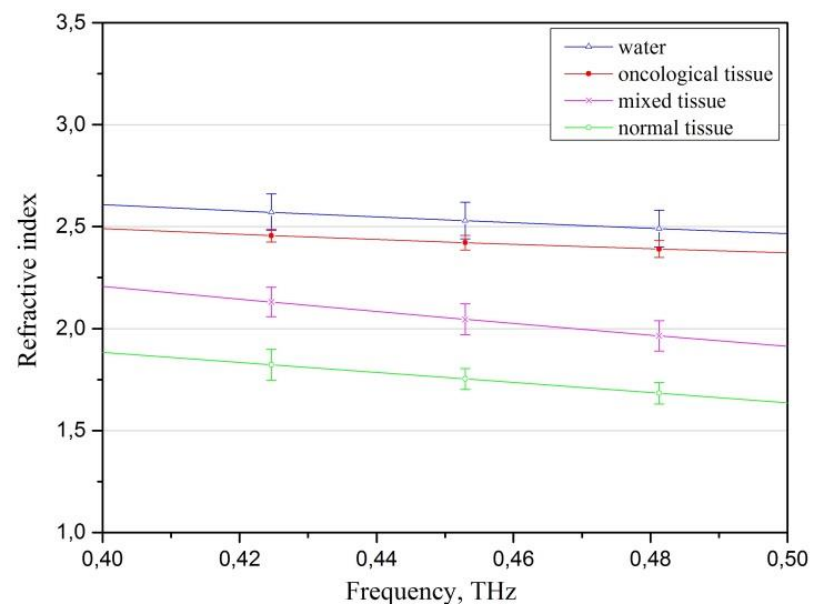

Fig. 4. The dispersion of real part of refractive indices of water, cancer, mixed and normal tissues in frequency range of $0.4-0.5 \mathrm{THz}$.

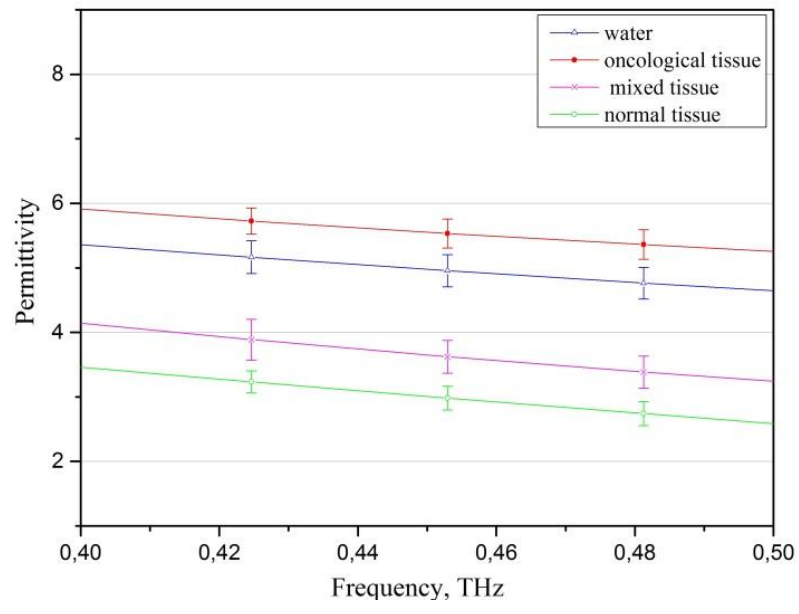

Fig. 5. The dispersion of real part of permittivities of water, cancer, mixed and normal tissues in frequency range of $0.4-0.5 \mathrm{THz}$.

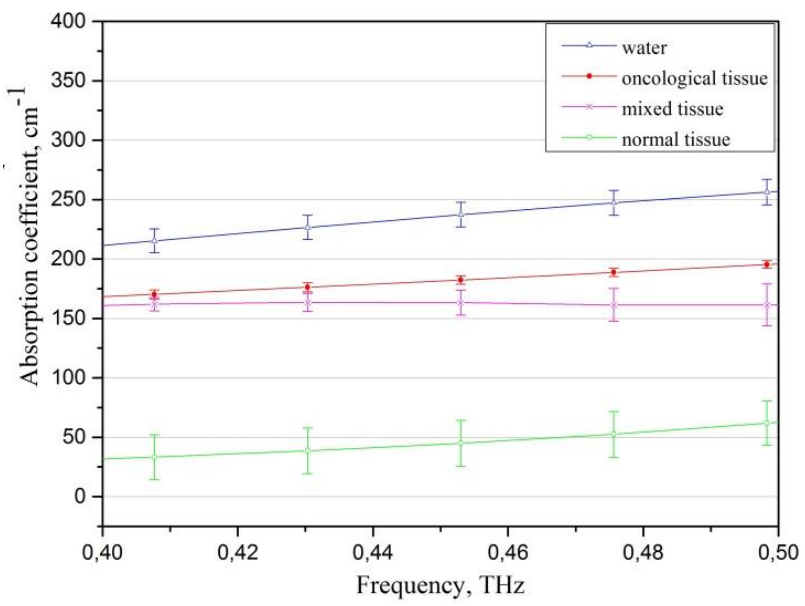

Fig. 6. The dispersion of absorption coefficient of water, cancer, mixed and normal tissues in frequency range of $0.4-0.5 \mathrm{THz}$. 
The dispersions of absorption coefficients (Fig. 6) for three investigated tissues and distilled water approve the theory [12] It is demonstrated that the absorption coefficient becomes lower from distilled water to normal tissue with decreasing of water molecules in water, oncological, mixed and normal tissues respectively.

\section{DISCUSSION}

The results of presented work clearly demonstrate pronounced differences in optical characteristics, such as refractive index, permittivity and absorption coefficient of human oncological,

mixed, and normal gastrointestinal tissue in a frequency range of $0.4-0.5 \mathrm{THz}$. According to the literature, optical parameters of tissue in terahertz spectrum are in strong relation with the water content of the studied sample. The character of $\mathrm{O}-\mathrm{H}$ boundaries determines the high absorbency of water in infrared and terahertz radiation areas and the highest absorption coefficient of the most hydrated tissues. It was shown that the water content of organs changes under the pathological conditions as a result of edema and increased vascularization [17].

\section{CONCLUSION}

As a result of this study a new promising in vivo method for gastric cancer diagnostic was described

\section{ACKNOWLEDGMENTS}

This work was financially supported by the Government of Russian Federation, Grant 074-U01.

This project has received funding from the European Union's Horizon 2020 research and innovation programme under the Marie Skłodowska-Curie grant agreement No 713606.

\section{REFERENCES}

[1] V.I. Chissov, V.V. Starinsky and G.V. Petrova, "Malignant neoplasms in Russia in 2010," Herzen Moscow Oncology Research Institute Press pp. 216, 2012.

[2] World Cancer Research Fund International, Cancer facts \& figures 2012. available from: http://www.wcrf.org/ .

[3] Cancer Statistics, available from: https://www.cancer.gov/aboutcancer/understanding/statistics .

[4] V.P. Wallace, D.A. Arnone, R.M. Woodward and R.J. Pye, "Biomedical applications of terahertz pulse imaging," 24th Annual Conference and the Annual Fall Meeting of the Biomedical Engineering Society Proceedings of the Second Joint EMBS/BMES Conference, pp. 2333-2334, 2002.

[5] Y. Sun, M. Y. Sy, Y.-X.J. Wang, A.T. Ahuja, Y.-T. Zhang and E. Pickwell-MacPherson, "A promising diagnostic method: Terahertz pulsed imaging and spectroscopy," World J Radiol; vol. 3(3), pp. 55-65, 2011.

[6] J.T. Kindt and C.A. Schmuttenmaer, "Far-infrared dielectric properties of polar liquids probed by femtosecond terahertz pulse spectroscopy," J. Phys. Chem., vol. 100, pp. 10373-9, 1996.

[7] A.R. Orlando and G.P. Gallerano, "Terahertz radiation effects and biological applications," J Infrared Millimeter Terahertz Waves, vol. 30, pp. 1308-1318, 2009.

[8] G. C. Trichopoulos and K. Sertel, "Polarimetric Terahertz Probe for Endoscopic Assessment of Malignancies," Proceedings of 2015 IEEE International Symposium on Antennas and Propagation \& USNC/URSI National Radio Science Meeting, pp. 730-731, 2015.
[9] R. Herrero, J. Parsonnet and E.R. Greenberg, "Prevention of Gastric Cancer," JAMA, vol. 312(12), pp. 1197-1198, 2014.

[10] C.H. Park, B. Kim, H. Chung, H. Lee, J.C. Park, S.K. Shin, S. K. Lee and Y.C. Lee, "Endoscopic Quality indicators for esophagogastroduodenoscopy in gastric cancer screening," Dig. Dis. Sci. Epub ahead of print, 2014.

[11] V.P. Wallace, A.J. Fitzgerald and E. Pickwell, "Terahertz pulsed spectroscopy of human basal cell carcinoma," Appl. Spectroscopy, vol. 60, pp. 1127-1133, 2006.

[12] A.J. Fitzgerald, V.P. Wallace, M. Jimenez-Linan, L. Bobrow, R. J. Pye, A.D. Purushotham, and D.D. Arnone, "Terahertz pulsed imaging of human breast tumors," Radiology, vol. 239(2), pp. 533-540, 2006.

[13] P.C. Ashworth, E. Pickwell-MacPherson, E. Provenzano, S.E. Pinder, A.D. Purushotham, M. Pepper, and V.P. Wallace, "Terahertz pulsed spectroscopy of freshly excised human breast cancer," Opt. Express, vol. 17(15), pp. 12444-12454, 2009.

[14] C.B. Reid, A. Fitzgerald, G. Reese, R. Goldin, P. Tekkis, P.S. O’Kelly, E. Pickwell-MacPherson, A.P. Gibson, and V.P. Wallace, "Terahertz pulsed imaging of freshly excised human colonic tissues," Phys. Med. Biol., vol. 56(14), pp. 4333-4353, 2011.

[15] E. Bombardieri, J. Buscombe, G. Lucignaniand and O. Schober, "Advances in Nuclear Oncology: Diagnosis and Therapy," Taylor \& Francis, Chap. 3, 2007.

[16] P.U. Jepsen, "Determining parameters of the dielectric function of a substance in aqueous solution by self-referenced reflection $\mathrm{THz}$ spectroscopy," US 8374800, 2013.

[17] S. Sy, S. Huang, Y.-X.J. Wang, J. Yu, A.T. Ahuja, Y.T. Zhang, and E. Pickwell-MacPherson, "Terahertz spectroscopy of liver cirrhosis: investigating the origin of contrast," Physics in medicine and biology, vol. 55(24), pp. 7587, 2010. 